\title{
MAGNETOHYDRODYNAMIC SIMULATION OF THE EVOLUTION OF BIPOLAR MAGNETIC REGIONS
}

\author{
S. T. WU AND C. L. YIN*
}

Center for Space Plasma and Aeronomic Research and Department of Mechanical Engineering The University of Alabama in Huntsville Huntsville, Alabama 35899 U.S.A.

\section{P. MCINTOSH AND E. HILDNER}

Space Environment Laboratory, National Oceanic and Atmospheric Administration, Boulder, Colorado 80303 U.S.A.

\begin{abstract}
It has been recognized that the magnetic flux observed on the solar surface appears first in low latitudes, and then this flux is gradually dispersed by super granular convective motions and meridional circulation. Theoretically, the magnetic flux transport could be explained by the interactions between magnetic fields and plasma flows on the solar surface through the theory of magnetohydrodynamics.

To understand this physical scenario, a quasi-three-dimensional, timedependent, MHD model with differential rotation, meridional flow and effective diffusion as well as cyclonic turbulence effects is developed. Numerical experiments are presented for the study of Bipolar Magnetic Regions (BMRs). When the MHD effects are ignored, our model produced the classical results (Leighton, Astrophys. J., 146, 1547, 1964). The full model's numerical results demonstrate that the interaction between magnetic fields and plasma flow (i.e., MHD effects), observed together with differential rotation and meridional flow, gives rise to the observed complexity of the evolution of BMRs.
\end{abstract}

\section{INTRODUCTION}

Leighton's model of sunspots and solar cycle in relation to the expansion and migration of unipolar (UM) and bipolar (BM) magnetic regions was published in 1964. Since then, a number of investigators (DeVore et al 1984; McIntosh and Wilson, 1985; Sheeley, et al 1985; Sheeley, and Devore, 1986; Wilson, 1986; Wilson and McIntosh, 1991; Wang and Sheeley, 1991) have extensively studied the magnetic flux transport in relation to the solar cycle by means of a modified Leighton model with additional physics. Recent rapid development of numerical simulation gives us the capability to study highly complex, nonlinear mathematical systems. Wang and Sheeley (1991) have presented a numerical simulation including differential rotation, supergranular diffusion, and a poleward surface flow (i.e., meridional flow) of the redistribution of magnetic flux 
erupting in the form of BMRs. They reproduced many of the observed properties of the Sun's large-scale field not encompassed by Babcock's (1961) or Leighton's (1964) model. Wilson and McIntosh (1991) compared observed evolutions of large-scale magnetic fields with simulated evolutions based on the kinematic model of Devore and Sheeley (1987). They concluded there must be significant contributions to the evolving patterns by non-random flux eruptions within the network structure, independent of active regions.

On the basis of magnetohydrodynamic theory, we understand that the nonlinear dynamical interactions between the plasma flow and magnetic field are essential to understand magnetic fiux transport. However, none of the previous investigations have invoked MHD to study this problem. In this paper, we present a quasi-three-dimensional, time-dependent, MHD model with differential rotation, meridional flow and effective diffusion as well as cyclonic turbulence to study magnetic evolution of BMRs. The mathematical model, initial and boundary conditions are presented in Section II. Numerical results and concluding remarks are given in Sections III and IV respectively.

\section{MATHEMATICAL MODEL, INITIAL AND BOUNDARY CONDITIONS}

The mathematical model appropriate for the physical scenario we described in the previous section can be expressed by a set of incompressible simulated MHD equations including differential rotation, meridional flow and turbulent diffusion. These governing equations are:

$$
\nabla \cdot \vec{u}=0
$$

$$
\begin{gathered}
\rho\left[\frac{\partial \vec{u}}{\partial t}+(\vec{u} \cdot \nabla)(\vec{u}-\vec{u} \prime)\right]= \\
-\nabla p+\frac{1}{4 \pi}(\nabla \times \vec{B}) \times \vec{B}+\vec{F}_{g}-2 \rho \vec{\omega}_{0} \times(\vec{u}-\vec{u} \prime) \\
-\rho \vec{\omega}_{0} \times\left[\vec{\omega}_{0} \times\left(\vec{r}-\vec{r}^{\prime}\right)\right]+\mu_{t} \nabla^{2}(\vec{u}-\vec{u} \prime) \\
\frac{\partial \vec{B}}{\partial t}=\nabla \times(\vec{u} \times \vec{B})+\lambda(\nabla \times \vec{B})+\kappa \nabla^{2} \vec{B}+\vec{S} \\
\frac{\partial p}{\partial t}+(\vec{u} \cdot \nabla) p=\Delta Q+(\gamma-1)\left[\frac{J^{2}}{\sigma_{t}}+\frac{\mu_{t}}{2}\left(\frac{\partial u_{i}}{\partial x_{k}}+\frac{\partial u_{k}}{\partial x_{j}}\right)^{2}\right]
\end{gathered}
$$

where $\rho$ is the plasma mass density, $\vec{u}$ the plasma flow velocity vector, $p$ the plasma thermal pressure, and $\vec{B}$, the magnetic induction vector, respectively. The other quantities are defined as follows; $\vec{\omega}_{0}$ is the angular velocity of solar differential rotation referring to the center of the solar coordinate system, that is given by empirical value as

$$
\vec{\omega}_{o}=\hat{i}_{\phi} \frac{\left(0.2337-0.04835 \cos ^{2} \theta_{o}\right)}{86400} \operatorname{arcsec~s}{ }^{-1}
$$

with $\theta_{o}$ being the latitude of the center of a sunspot (or active region). The $\vec{u} \prime$ is the prescribed background plasma velocity field including differential rotation and meridional flow which is given by 


$$
\vec{u} \prime=u \prime_{r} \hat{i}_{r}+u \prime_{\theta} \hat{i}_{\theta}+u \prime_{\phi} \hat{i}_{\phi}
$$

with $u_{r}$ being the radial component velocity on the solar surface, $u_{\theta}^{\prime}$ is the velocity of the meridional flow which is chosen to be $u_{\theta} l=0.015 / \sin \theta$ for the present study and $u_{\phi}^{\prime}$ is the velocity due to the differential rotation relative to the rotating coordinate system, that is

$$
u \prime_{\phi}=\frac{0.04835\left(\cos ^{2} \theta_{o}-\cos ^{2} \theta\right) r \sin \theta}{86400} \quad \operatorname{arcsec~s}^{-1}
$$

$\vec{r} \prime$ is the dummy position vector referring to the location of the sunspot (or active region), $\vec{F}_{g}$ is the gravitational force of the sun, $\kappa$ is the effective diffusivity and $\lambda$ represents the coefficient of the cyclonic turbulence, $\vec{S}$ is the additional source terms such as emerging flux etc., the $\mu_{t}$ is the turbulent viscosity.

Finally the term $\Delta Q$ represents the heat source due to the initial dynamics of the quiet photospheric motion such that

$$
\Delta Q=(\vec{u} \prime \cdot \nabla) p_{o}-(\gamma-1)\left[\frac{J_{0}^{2}}{\sigma_{t}}+\frac{\mu_{t}}{2}\left(\frac{\partial u t_{i}}{\partial x_{k}}+\frac{\partial u t_{k}}{\partial x_{i}}\right)^{2}\right]
$$

This term is prescribed to assure the self-consistency of the MHD solution, namely, the initial dynamical equilibrium of the solar atmosphere is obtained.

This set of MHD equations differs from those of usual first principle MHD theory. These differences arise from additional physics we have included in this formulation. For example, the additional terms in Equation (2) represent the inertia centrifugal force (i.e. $2 \rho \omega_{0} \times(\vec{u}-\vec{u} \prime)$ ), the coriolis force (i.e. $2 \rho \vec{\omega}_{0} \times$ $\left(\vec{\omega}_{0} \times(\vec{r}-\vec{r} f)\right)$ ) and effective diffusion due to random motion of the granules or the super-granules. The additional term in Equation (3) represents the cyclonic turbulence (i.e. $\lambda(\nabla \times \vec{B})$ ).

Ideally, we should solve this set of equations in full three dimensions. However, this is costly and unnecessary, as we are interested only in exploring the fundamental physical processes rather than in simulating the realistic situation on the sun. Consequently, we prescribe the behavior of the radial derivatives of velocity and magnetic field on the basis of the mathematical convenience. These functions are:

$$
\begin{gathered}
\vec{B}=B_{r}(r) B_{r}(\theta, \phi) \hat{i}_{r}+B_{\theta}(r) B_{\theta}(\theta, \phi) \hat{i}_{\phi}+B_{\phi}(r) B_{\phi}(\theta, \phi) \hat{i}_{\phi} \\
\vec{u}=u_{r}(r) u_{r}(\theta, \phi) \hat{i}_{r}+u_{\theta}(r) u_{\theta}(\theta, \phi) \hat{i}_{\theta}+u_{\phi}(r) u_{\phi}(\theta, \phi) \hat{i}_{\phi}
\end{gathered}
$$

with

$$
\begin{aligned}
& \frac{\partial B_{r}}{\partial r}=-\left(\frac{2}{r}+\frac{a}{H_{0}}\right) B_{r} \\
& \frac{\partial B_{\theta}}{\partial r}=-\left(\frac{1}{r}+\frac{a}{H_{0}}\right) B_{\theta} \\
& \frac{\partial B_{\phi}}{\partial r}=-\left(\frac{1}{r}+\frac{a}{H_{0}}\right) B_{\phi}
\end{aligned}
$$




$$
\begin{gathered}
\frac{\partial u_{r}}{\partial r}=-\frac{2}{r} u_{r} \\
\frac{\partial u_{\theta}}{\partial r}=\frac{1}{r} u_{\theta} \\
\frac{\partial u_{\theta}}{\partial r}=\frac{1}{r} u_{\phi}
\end{gathered}
$$

Substituting equation (12) and (13) into equation (2) by setting $u_{r} \equiv u_{r}^{\prime}$, we have

$$
\begin{array}{r}
\frac{\partial p}{\partial r}=\frac{p}{r}\left[u_{\theta}\left(u \theta-u_{\theta}^{\prime}\right)+u_{\phi}\left(u_{\phi}-u_{\phi^{\prime}}\right)\right]+2 p \omega_{o} \sin \theta\left(u_{\phi}-u_{\phi^{\prime}}\right)-\rho g+ \\
\frac{1}{4 \pi}\left[\frac{B_{\theta}}{r}\left(\frac{\partial B_{\theta}}{\partial \theta}-\frac{\partial\left(r B_{\theta}\right)}{\partial r}\right)\right]-\frac{B_{\phi}}{r}\left(\frac{\partial}{\partial r}\left(r B_{\phi}\right)\right)-\frac{1}{\sin \theta} \frac{\partial B_{r}}{\partial \phi}
\end{array}
$$

where $H_{0}$ is the depth of the sunspot (i.e. $\sim 2 \times 10^{4} \mathrm{~km}$ ) and $a$ is an arbitrary constant which is the measure of the decreasing rate of field strength against height.

Using these relationships, the computation reduces to a calculation on the solar surface. That is why we called our model a quasi-three-dimensional, timedependent MHD model.

To assure self-consistency of the numerical simulation of the evolutionary MHD processes, proper initial and boundary conditions are needed. We obtain our initial conditions by assuming the solar atmosphere is in dynamical equilibrium. Thus, these initial conditions are:

$$
\begin{aligned}
\frac{1}{r \sin \theta} \frac{\partial}{\partial \theta}\left(B_{\theta 0} \sin \theta\right)+\frac{1}{r \sin \theta} \frac{\partial}{\partial \phi}\left(B_{\phi o}\right) & =\frac{a}{H_{0}} B_{r 0} \\
\frac{B_{\theta 0}}{B_{\phi 0}} & =-\tan \alpha
\end{aligned}
$$

with

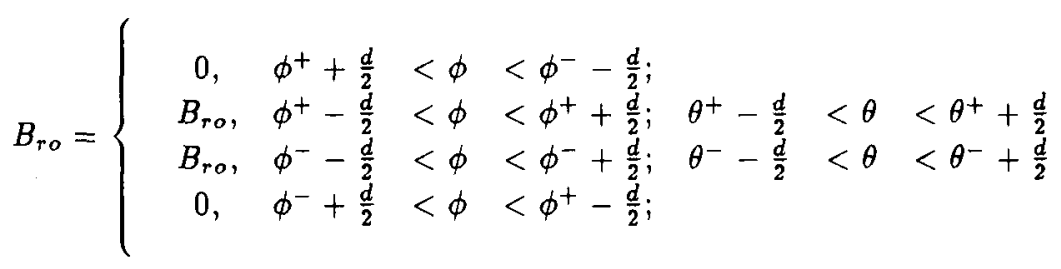

where $\left(\phi^{+}, \theta^{+}\right)$and $\left(\phi^{-}, \theta^{-}\right)$represent the coordinates of sunspots with positive and negative polarity respectively and $d$ represents the diameter of the sunspots. 
(ii) Initial velocity field is given by

$$
\begin{array}{r}
u_{r}=u_{r} \prime=0 \text { or } 0.1 \mathrm{kms}^{-1} \\
u_{\theta}=u_{\theta}^{\prime}=\frac{0.015}{\sin \theta}, \quad \mathrm{kms}^{-1} \\
u_{\phi}=u_{\phi}^{\prime}=\omega_{0}(\theta) r \sin \theta \quad \mathrm{kms}^{-1}
\end{array}
$$

(iii) Initial pressure field is

$$
\begin{array}{r}
\frac{1}{r} \frac{\partial p_{0}}{\partial \theta}=\frac{1}{4 \pi}\left[\frac{B_{\phi 0}}{1 \sin \theta}\left(\frac{\partial B_{\theta 0}}{\partial \phi}-\frac{\partial}{\partial \theta}\left(B_{\phi} \sin \theta\right)\right)\right] \\
-\frac{B_{r 0}}{r}\left(\frac{a r}{H_{0}} B_{\theta 0}+\frac{\partial B_{r 0}}{\partial \theta}\right) \\
\frac{1}{r \sin \theta} \frac{\partial p_{0}}{\partial \phi}=\frac{1}{4 \pi}\left[\frac{B_{\theta 0}}{r \sin \theta}\left(\frac{\partial}{\partial \theta}\left(B_{\phi} \sin \theta\right)-\frac{\partial B_{\theta 0}}{\partial \phi}\right)\right. \\
\left.-\frac{B_{r 0}}{r}\left(\frac{a r}{H_{0}} B_{\phi 0}+\frac{1}{\sin \theta} \frac{\partial B_{r 0}}{\partial \phi}\right)\right]
\end{array}
$$

In addition, the $B_{r \phi}, B_{\theta 0}$ and $B_{\phi 0}$ are given to satisfy the solenoidal condition (i.e. $\nabla \cdot \vec{B}=0$ ).

The boundary conditions employed here are computational boundary conditions which simply are the forward differencing linear extrapolation.

\section{NUMERICAL SIMULATION RESULTS}

The computational domain for the simulation is set as a rectangular region with 51 grids in the carrington longitude $(\phi)$ and 35 grids in the latitudinal direction $(\theta)$ in which we have chosen 0.5 degree for each grid in this study. It is assumed that the differential rotation and meridional flow velocity depend upon latitude only. Therefore, the prescribed flow pattern within the computational domain will depend on the domain's latitude but not on its longitude.

Before we carry out this simulation study, we need to know two important coefficients: effective diffusivity $(\kappa)$ and cyclonic turbulence $(\lambda)$. We know that the range of values of effective diffusivity is quite wide, for example, $\kappa=160-$ $300 \mathrm{~km}^{2} \mathrm{~s}^{-1}$ given by Parker (1979); Leighton's value of $\kappa$ is $800-1600 \mathrm{~km}^{2} \mathrm{~s}^{-1}$ (Leighton, 1964); DeVore et al (1985) selected $\kappa=300 \mathrm{~km}^{2} \mathrm{~s}^{-1}$ for their study. Wang (1988) derived a value of $\kappa$ being $100-150 \mathrm{~km}^{2} \mathrm{~s}^{-1}$ on the basis of observations. The purpose of this study is to learn the fundamental physical processes due to the simulated MHD effects (i.e. photospheric dynamo) and is not to simulate a particular event. Therefore, we simply choose values within these ranges for this study. The value of cyclonic turbulence is chosen according to the scale law $(\lambda<\kappa / L)$, where $L$ is the characteristic length of sunspot, it is chosen to be $6000 \mathrm{~km}$ for the present study.

The first issue which we shall examine is how the simulated MHD process (i.e. photospheric dynamo) will effect the Leighton's results (1964). To achieve 
this purpose, we simply reduce the present set of governing equations into a single diffusion type equation of Leighton's theory which is expressed by Eq. (3) with $\lambda=0$. To perform such a simulation, we set up a bipolar magnetic region with strength $\pm 1800 G$ and two degrees apart, then, allow the mathematical system to evolve. The $B_{r}$ contours for $\kappa=120 \mathrm{~km}^{2} \mathrm{~s}^{-1}$ at time $240 \mathrm{hrs}$ after introduction of the bipolar magnetic region (BMR) are shown in Figure 1a which resembles the classical Leighton's results. As we have pointed out earlier, these results simply represent the magnetic field transport due to effective diffusion and differential rotation. Figure $1 \mathrm{~b}$ shows the simulation results using the full set of governing equations with differential rotation and $\kappa$ equal $120 \mathrm{~km}^{2} \mathrm{~s}^{-1}$ at 240 hrs after introduction of the BMR. It shows the interaction between the plasma flow and magnetic fields. In such a case, the photospheric dynamo process sets in. Significant differences between Fig.1a and $1 \mathrm{~b}$ are evident; (i) with MHD effects the simplicity of magnetic field strength contours disappeared, (ii) the magnetic field has migrated significantly in longitudinal direction, because we have ignored meridional flow in this calculation and (iii) the MHD effects lead to highly sheared neutral line.

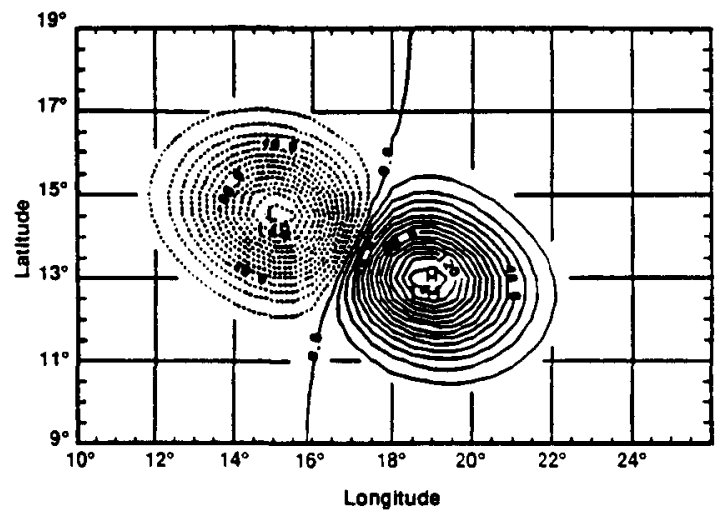

(a)

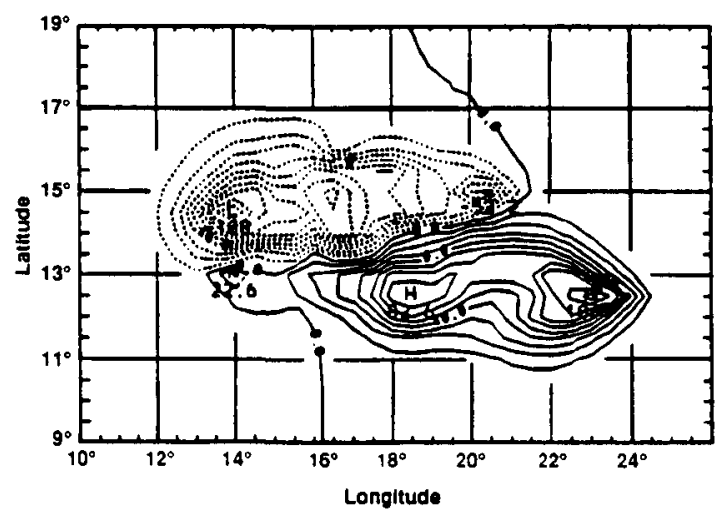

(b)

Fig. 1 The radial magnetic field strength $\left(B_{r}\right)$ contours at $240 \mathrm{hrs}$ after introduction of the BMR without (a) and with (b) simulated MHD effects. 


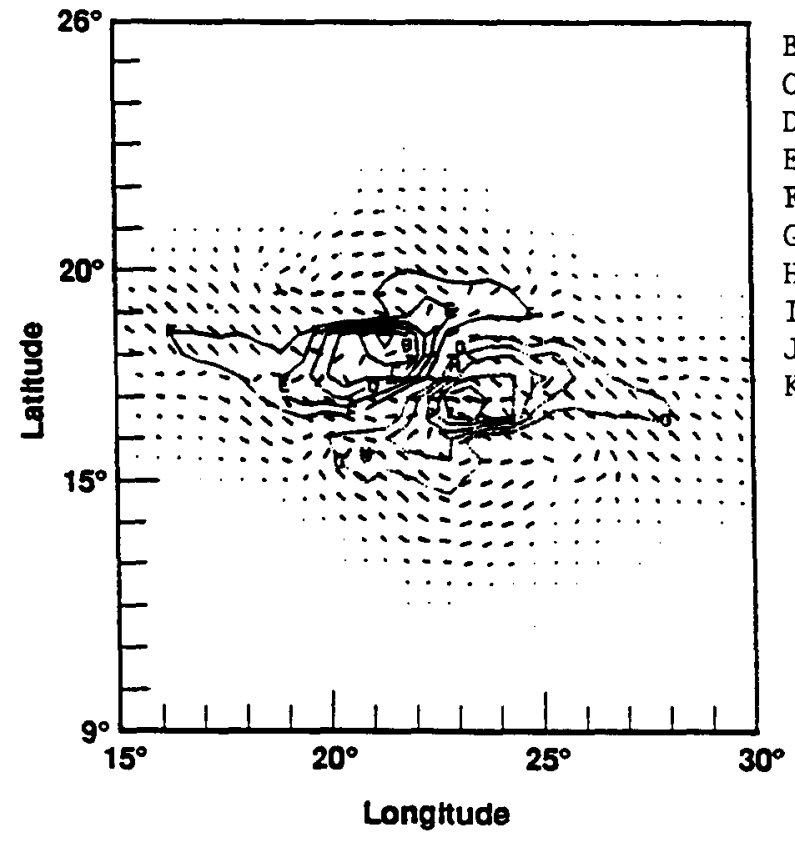

$\begin{array}{lll}B & (180 & G) \\ C & (145 & G) \\ D & (110 & G) \\ E & (75 & G) \\ F & (40 & G) \\ G & (-40 & G) \\ H & (-75 & G) \\ I & (-110 & G) \\ J & (-145 & G) \\ K & (-180 & G)\end{array}$

(a)

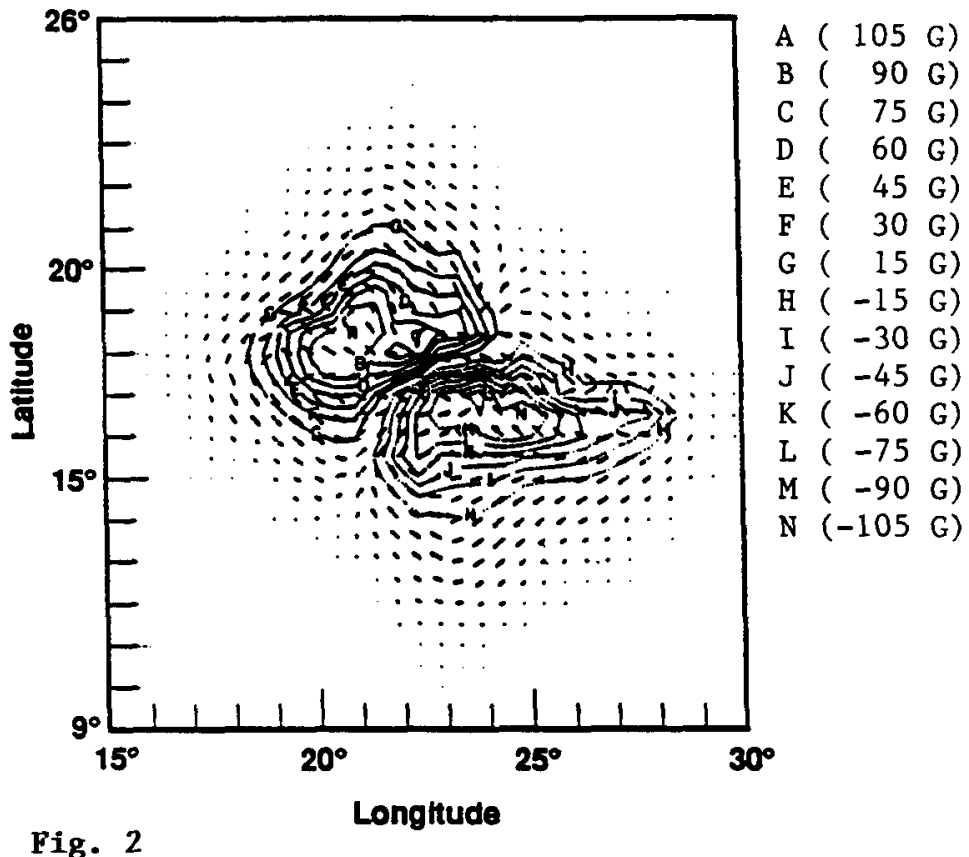

(b)

The radial magnetic field strength $\left(B_{r}\right)$ contours and transverse magnetic field at 120 hrs after introduction of the BMR without meridional flow (a) and with meridional flow (b) using the full set of simulated MHD equations. 
Figure 2 shows the effects of the meridional flow on the magnetic field transport, Figure $2 \mathrm{a}$ exhibits the $B_{r}$ strength contours and transverse field for $\kappa=200 \mathrm{~km}^{2} \mathrm{~s}^{-1}$ and $\lambda=0.0125 \mathrm{~km} \mathrm{~s}^{-1}$ without a meridional flow and Figure $2 \mathrm{~b}$ shows the same case with meridional flow. It may note that the meridional flow produced a slightly poleward movement of the BMR.

Up to now, we have shown the computed evolution of the morphology of the BMR with and without MHD effects. It is interesting to understand the energy evolution during these processes. In Figure 3, we show the magnetic energy evolution for the cases with and without background velocity effects. Figure 3a shows the normalized magnetic energy (i.e., $E_{m}(t) / E_{m}(0)$ ) in the absence of prescribed differential rotation and meridional flow for the case when only diffusion and cyclonic turbulence are included. We notice that the normalized magnetic energy $E_{m}(t) / E_{m}(0)$ of the BMR through effective diffusion $\left(\kappa=200 \mathrm{~km}^{2} \mathrm{~s}^{-1}\right)$ is decreasing almost linearly with time as represented by curve $B$. If both effective diffusion and cyclonic turbulence $\lambda=0.25 \mathrm{~km} \mathrm{~s}^{-1}$ are considered, $E_{m}(t) / E_{m}(0)$ decreased a little more slowly than curve $A$. Curve $C$ represents the case for $c y$ clonic turbulence only and $E_{m}(t) / E_{m}(0)$ shows a slight increase with time. This clearly informs us that the photosphere dynamo can be an energy production source. In Figure $3 \mathrm{~b}$ we test the influence of effective diffusion (loss) and cyclonic turbulence (source) in the presence of differential rotation and meridional flow on the evolution of the normalized magnetic energy. Curve A represents the case for $\kappa=\lambda=0$; it gives $E_{m}(t) / E_{m}(0)$ being constant, this informs us that this simulation model has maintained dynamical equilibrium throughout the process and it validates the accuracy of the simulation model. Curves $\mathrm{B}$ and $\mathrm{C}$ are for $\lambda=0$ and $\kappa=100 \mathrm{~km}^{2} \mathrm{~s}^{-1}$ and $200 \mathrm{~km}^{2} \mathrm{~s}^{-1}$, respectively. It is apparent that, when the effective diffusivity increases, the decay rate of normalized magnetic energy increases. Curves $D$ and $E$ in Figure $3 b$ show the evolution of normalized magnetic energy for $\kappa=0$ with $\lambda=0.75 \mathrm{~km} \mathrm{~s}^{-1}$ and $0.1 \mathrm{~km} \mathrm{~s}^{-1}$, respectively. It is immediately noticed that the growth rate of normalized magnetic energy increased with increasing $\lambda$. From these studies, we realize that effective diffusion and cyclonic turbulence are competing physical mechanisms for the magnetic energy evolution in the magnetic flux transport process of a BMR. In Figure 4, we present the normalized magnetic energy $v s$ time for a fixed value of cyclonic turbulence $\left(\lambda=0.025 \mathrm{~km} \mathrm{~s}^{-1}\right)$ and effective diffusivity 0,100 and $200 \mathrm{~km}^{2} \mathrm{~s}^{-1}$ respectively. These results clearly show how these two competing mechanisms work; when $\kappa=0$, the magnetic energy will increase with time but when effective diffusivity dominates cyclonic turbulence, then the magnetic energy of BMR decays.

\section{CONCLUDING REMARKS}

The purpose of this study has been to show how a simulated magnetohydrodynamic (MHD) process which incorporates to the photospheric dynamo might affect the evolution of a bipolar magnetic region (BMR) and lead to the complex active region on the solar surface. To accomplish this purpose, a quasithree-dimensional, time-dependent MHD model was developed. For the present exploratory simulation study, we realize that there are two major physical mechanisms which interplay during the evolutionary process of a BMR, and could 


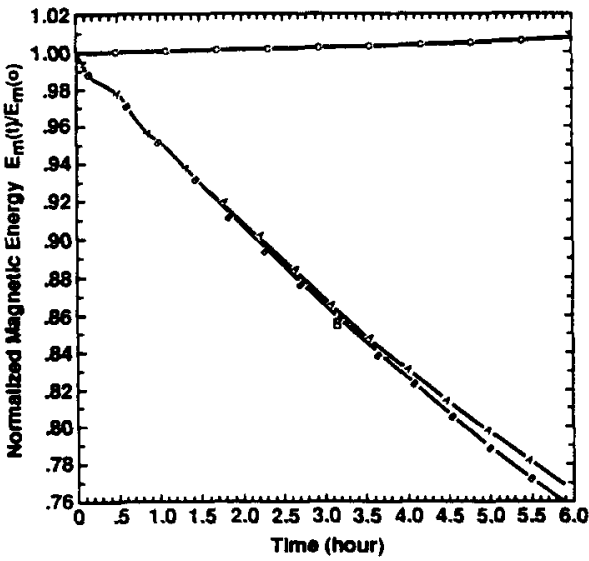

(a)

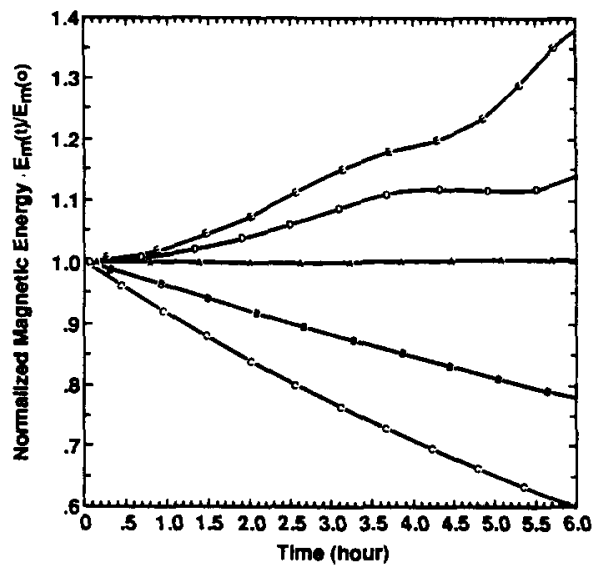

(b)

Fig. 3

(a) The evolution of the normalized magnetic energy without MHD effect: A $\left(\kappa=200 \mathrm{~km}^{2} \mathrm{~s}^{-1}, \lambda=0.025 \mathrm{~km} \mathrm{~s}^{-1}\right), \mathbf{B}\left(\kappa=200 \mathrm{~km}^{2} \mathrm{~s}^{-1}, \lambda=\right.$ $\left.0 \mathrm{~km} \mathrm{~s}^{-1}\right)$, and $\mathbf{C}\left(\kappa=0, \lambda=0.025 \mathrm{~km} \mathrm{~s}^{-1}\right)$. (b) The evolution of the normalized magnetic energy with MHD effect: $\mathbf{A}(\kappa=0, \lambda=0), \mathbf{B}$ $\left(\kappa=100 \mathrm{~km}^{2} \mathrm{~s}^{-1}, \lambda=0\right), \mathbf{C}\left(\kappa=200 \mathrm{~km}^{2} \mathrm{~s}^{-1}, \lambda=0\right), \mathbf{D}(\kappa=0, \lambda=$ $\left.0.075 \mathrm{~km} \mathrm{~s}^{-1}\right), \mathbf{E}\left(\kappa=0, \lambda=0.1 \mathrm{~km} \mathrm{~s}^{-1}\right)$.

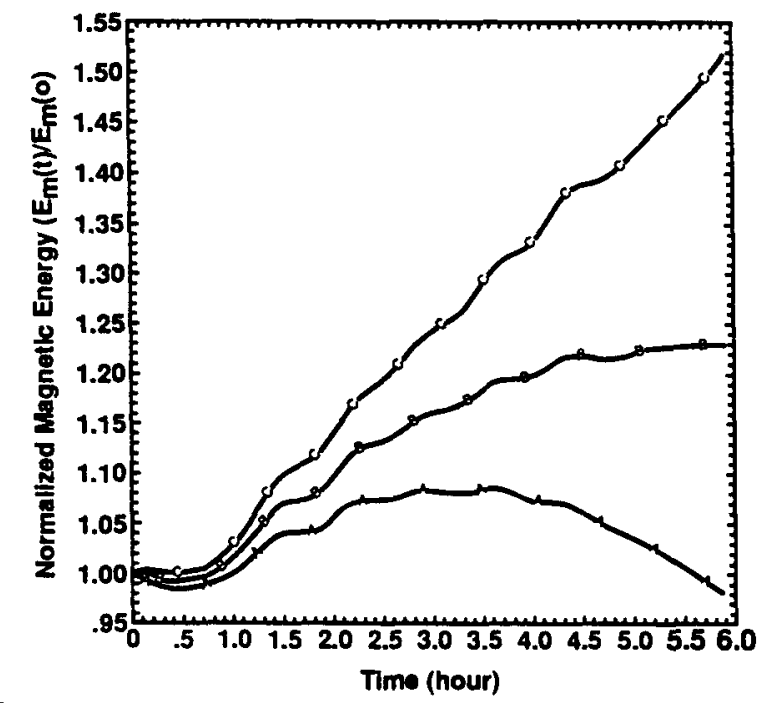

Fig. 4

The evolution of normalized magnetic energy with MHD effect and varjous magnitude of effective diffusion: $A\left(\kappa=200 \mathrm{~km}^{2} \mathrm{~s}^{-1}\right), \mathbf{B}(\kappa=$ $\left.100 \mathrm{~km}^{2} \mathrm{~s}^{-1}\right)$, and $\mathbf{C}(\kappa=0)$, at fixed value of cyclonic turbulence $\left(\lambda=0.025 \mathrm{~km} \mathrm{~s}^{-1}\right)$. 
explain the magnetic flux transport on the solar surface. These two physical mechanisms are the effective diffusivity and cyclonic turbulence with simulated MHD process which triggers the photosphere dynamo. The most interesting results from the present simulation can be summarized as follows: (i) If the simulated MHD process is appropriate, the decay of a BMR will create more complex structures because the nonlinear interactions exist between the plasma flows and background magnetic fields and (ii) the magnetic energy of the BMR could be increased if the cyclonic turbulence process dominates. In summary, we may conclude that MHD interactions are important and needed in the study of magnetic field transport.

\section{ACKNOWLEDGEMENT}

The work by STW and CLY was supported by a NASA Headquarters grant NAGW-9 and NOAA (50RANR000104).

*Permanent Address: Purple Mountain Observatory, Nanjing, PRC

\section{REFERENCES}

1. DeVore, C. Richard, Sheeley, N. R., Jr., Boris, J. P., Young, R. T., Jr., and Harvey, K. L.: 1984, Solar Phys. 92, 1.

2. DeVore, C. R., Sheeley, N. R., Jr., Boris, J. P., Young, R. T. Jr., and Harvey, K. L.: 1985 Solar Phys. 102, 41.

3. DeVore, C. R., and Sheeley, N. R., Jr.: 1987 Solar Phys. 108, 47.

4. Leighton, Robert B.: 1964, Ap.J. 140, 1547.

5. McIntosh, P. S. and Wilson, P. R.: 1985, Solar Phys. 97, 59.

6. Parker, E. N., Cosmic Magnetic Fields, Oxford University Press, England, p. $509,1979$.

7. Sheeley, N. R., Jr., DeVore, C. R., and Boris, J. P.: 1985 Solar Phys. 98, 219.

8. Sheeley, N. R. Jr., and DeVore, C. R.: 1986, Solar Phys. 103, 203.

9. Wang, H.: 1988, Solar Phys. 116, 1.

10. Wang, Y.-M. and Sheeley, N. R., Jr.: 1991,Ap. J. 375, 761.

11. Wilson, P. R.: 1986, Solar Phys. 106, 1.

12. Wilson, P. R. and McIntosh, P. S.: 1991 Solar Phys. 136, 221. 